\title{
Faint Fuzzy Star Clusters in NGC 1023 as Remnants of Merged Star Cluster Complexes
}

\author{
R.C. Brüns and P. Kroupa \\ Argelander-Institut für Astronomie, Universität Bonn, Auf dem Hügel 71, D-53121 Bonn, \\ Germany \\ rcbruens@astro.uni-bonn.de, pavel@astro.uni-bonn.de \\ and \\ M. Fellhauer \\ Departamento de Astronomía, Universidad de Concepción, Casilla 160-C, Concepción, Chile \\ mfellhauer@astro-udec.cl
}

\begin{abstract}
In the lenticular galaxy NGC 1023 a third population of globular clusters (GCs), called faint fuzzies (FFs), was discovered next to the blue and red GC populations by Larsen \& Brodie. While these FFs have colors comparable to the red population, the new population is fainter, larger $\left(R_{\mathrm{eff}}>7 \mathrm{pc}\right)$ and, most importantly, shows clear signs of co-rotation with the galactic disk of NGC 1023. We present N-body simulations verifying the hypothesis that these disk-associated FFs are related to the young massive cluster complexes (CCs) observed by Bastian et. al in M51, who discovered a massradius relation for these CCs. Our models have an initial configuration based on the observations from M51 and are placed on various orbits in a galactic potential derived for NGC 1023. All computations end up with a stable object containing 10 to $60 \%$ of the initial CC mass after an integration time of 5 Gyr. A conversion to visual magnitudes demonstrates that the resulting objects cover exactly the observed range for FFs. Moreover, the simulated objects show projected half-mass radii between 3.6 and $13.4 \mathrm{pc}$, in good agreement with the observed FF sizes. We conclude that objects like the young massive CCs in M51 are likely progenitors of the FFs observed in NGC 1023.
\end{abstract}

Subject headings: globular clusters: general — galaxies: individual (NGC 1023, M 51) - methods: n-body simulations — galaxies: star clusters — galaxies: evolution

\section{INTRODUCTION}

A large number of galaxies show a bimodal color distribution of their globular cluster (GC) systems, indicating a metal poor blue and a metal rich red GC system (West et al. 2004, and references 
therein). Larsen \& Brodie (2000) studied the GC system of the nearby (9.8 Mpc, Ciardullo et al. 2002) lenticular galaxy NGC 1023 and discovered a clear bimodal color distribution. The average effective radius of the blue GCs in NGC 1023 is about 2.0 pc, while the average radius of red compact GCs is slightly smaller $\left(R_{\text {eff }} \sim 1.7 \mathrm{pc}\right.$ ), as observed also for other galaxies (e.g. Larsen et al. 2001). The sizes for the blue clusters are as large as $R_{\text {eff }}=7 \mathrm{pc}$, while the sizes of the red clusters extend up to $R_{\text {eff }}=18 \mathrm{pc}$. The objects larger than $7 \mathrm{pc}$ are generally fainter than the red compact objects. The spatial distribution of these objects appears to be associated with the lenticular disk of NGC 1023. Due to their faint and extended appearance this new star cluster population was called "Faint Fuzzies" (FFs).

Brodie \& Larsen (2002) performed spectroscopic observations of the GCs in NGC 1023 and found that the FFs show a clear sign of co-rotation with the galactic disk, while the compact red GCs show no sign of rotation, suggesting that the FFs are indeed a separate population associated with the disk of NGC 1023. They averaged all FF spectra to achieve a high enough signal-to-noise ratio to estimate the age of these objects that appears to be older than 7-8 Gyr. Burkert et al. (2005) further analyzed the distribution and radial velocities of the FFs in NGC 1023 and suggested that they were the remnants of a past gravitational interaction forming a ring-like structure. Further observations also discovered FFs in the lenticular galaxy NGC 3384 (Brodie \& Larsen 2002), the dwarf irregular galaxy NGC 5195 (Lee et al. 2005), a number of galaxies of the Virgo cluster (Peng et al. 2006), and the lenticular galaxy NGC 1380 (Chies-Santos et al. 2007).

Observations have shown that young star clusters are often not isolated, but are part of larger structures called cluster complexes (CCs). Bastian et al. (2005) observed massive young star cluster complexes in the disk of the spiral galaxy M51 and found a scaling relation between the masses and the radii of the CCs that is comparable to the relation of the progenitor giant molecular clouds. This relation constrains the parameter space for the CC models in our simulations.

Kroupa (1998) studied the dynamical evolution of CCs observed to be forming in the interacting Antennae galaxies, and Fellhauer \& Kroupa (2002) performed the first N-body simulations indicating that FFs can be formed by merging of star clusters in CCs. The work presented in this paper is a follow-up study of these early computations. The simulations were performed to test the hypothesis that the FFs are the remnants of the young massive CCs as observed by Bastian et al. (2005) in M51.

In Section 2, we describe the method and the parameters used for the calculations. In Section 3. we present the results of the simulations, which will be discussed in Section 4 . 


\section{NUMERICAL SIMULATIONS}

\section{1. $\quad$ Method}

The simulations were performed with the particle-mesh code SUPERBOX (Fellhauer et al. 2000). The code solves the Poisson equation on a system of Cartesian grids. The code has a hierarchical grid structure. The local universe is covered by a fixed coarse grid which contains the orbit of the CC around the center of NGC 1023. The CC orbits in an analytical galactic potential (disk+bulge+halo). In order to get good resolution of the star clusters two grids with high and medium resolution are focused on each star cluster following their trajectories. The individual high resolution grids cover an entire star cluster, whereas the medium resolution grids of every star cluster embed the whole initial CC. All grids contain $64^{3}$ grid cells. The coordinate system is chosen such that the disk of NGC 1023 lies in the x-y-plane.

\subsection{Basic Parameters}

\subsubsection{An Estimate of the Gravitational Potential of NGC1023}

NGC 1023 is a disk-dominated SB0-galaxy at a distance of 9.8 Mpc (Ciardullo et al. 2002) in the constellation Perseus. It shows a well behaved rotation in the inner part, while the observed radial velocities in the outer regions appear to be more random (Noordermeer et al. 2008). This behavior is most likely related to a past interaction, which transformed the galaxy into an SB0 lenticular galaxy. Noordermeer et al. (2008) argue that the photometry of the disk is consistent with a passive fading since 1 Gyr after the galaxy lost its gas.

However the exact rotation curve is not required for the simulations because a good guess of the potential is sufficient to study the evolution of CCs 5 to 8 Gyr ago. In our simulations NGC 1023 is represented by an analytical potential, which consists of a disk, a bulge, and a halo component. For objects moving on circular orbits within the galactic plane, the results of the simulations depend mainly on the total force at a given distance, but very weakly on the exact choice of bulge, disk, and halo parameters. We use the observed velocities in the center of the galaxy from Simien \& Prugniel (1997) to constrain the rotation curve in the region of $R_{\text {gal }}<4 \mathrm{kpc}$ and assume a flat rotation curve for the outer part (Fig. 11).

The disk is modeled by a Miyamoto-Nagai potential (Mivamoto \& Nagai 1975)

$$
\Phi_{d i s k}(R, z)=-\frac{G M_{d}}{\sqrt{R^{2}+\left(a_{d}+\sqrt{z^{2}+b_{d}^{2}}\right)^{2}}},
$$

with $M_{\mathrm{d}}=5.5 \cdot 10^{10} \mathrm{M}_{\odot}, a_{\mathrm{d}}=2.8 \mathrm{kpc}$, and $b_{\mathrm{d}}=0.2 \mathrm{kpc}$. 
The bulge is represented by a Hernquist potential (Hernquist 1990)

$$
\Phi_{\text {bulge }}=-\frac{G M_{b}}{r+a_{b}}
$$

with $M_{\mathrm{b}}=0.7 \cdot 10^{10} \mathrm{M}_{\odot}$, and $a_{\mathrm{b}}=1.1 \mathrm{kpc}$. The halo is a logarithmic potential

$$
\Phi_{\text {halo }}(r)=\frac{1}{2} v_{0}^{2} \ln \left(r^{2}+r_{\text {halo }}^{2}\right),
$$

with $v_{0}=170 \mathrm{~km} \mathrm{~s}^{-1}$, and $r_{\text {halo }}=5.0 \mathrm{kpc}$.

For this project, we neglected heating sources from non-axisymmetric components like Giant Molecular Clouds (GMC) and spiral arms. This is a good approximation for compact objects with masses of about $10^{5} \mathrm{M}_{\odot}$. Gieles et al. (2006) demonstrated that star clusters with such masses cannot be destroyed by individual encounters with GMCs, if the extended nature of GMCs is taken into account. Close encounters between GMCs and massive star clusters act as tidal heating on the clusters. The corresponding disruption time is much larger than the integration time of the simulations presented in this paper. Gieles et al. (2007) analyzed the effect of spiral arms passages on the evolution of star clusters and found for massive clusters as considered in this paper a disruption time of the order 100 Gyr.

\subsubsection{Initial Configuration of the Cluster Complexes}

Bastian et al. (2005) derived a mass-radius relation for the CCs in M51 that constrains the parameter space for our CC models. They found $11 \mathrm{CCs}$ with ages younger than $10 \mathrm{Myr}$ in the disk of M51 that have sizes between $85-240 \mathrm{pc}$, and cover a mass range of $0.3-3 \cdot 10^{5} \mathrm{M}_{\odot}$. Most of these CCs show a massive concentration of clusters potentially merging in their centers and a couple of isolated clusters in their vicinity (see fig. 2 and 3 in Bastian et al. 2005). The size of a $\mathrm{CC}$ is defined as the point where the measured color is equal to that of the background stars in the disk. This radius corresponds to the cutoff radius of our models. The spatial distribution of the CCs is connected to the spiral arms of the disk. Most complexes are found at the outer edges of the spiral arms. The CCs show a mass-radius relation comparable to the one of the progenitor giant molecular clouds.

We choose the parameters of CCs in the simulations such that the models cover the two extremes (CC_H and CC_L) and a point in-between (CC_M) of the Bastian relation (Fig. 2).

The star clusters building up the CCs in our simulations are Plummer spheres (Plummer 1911; Kroupa 2008). The density distribution of the spheres is truncated at $R_{\text {cut }}=5 R_{\mathrm{pl}}$. Each star cluster consists of $N_{0}^{\mathrm{SC}}=100000$ particles. The CC models are diced according to a Plummer distribution with masses and radii taken from the Bastian relation (Fig. 2). Our models resemble the CCs observed by Bastian et al. (2005) with a high density concentration in their centers. 
Bastian et al. (2005) estimated a very high star formation efficiency within the CCs, using the CO-to- $\mathrm{H}_{2}$ conversion factor for M51 from Boselli et al. (2002) and a Salpeter IMF (Salpeter 1955). Due to the high star formation efficiency, gas expulsion has only little influence on the evolution of the merging CCs (Fellhauer \& Kroupa 2005). Consequently, the distorting effect of gas expulsion was not considered in the simulations.

\section{RESULTS}

We have carried out 25 different numerical simulations to study the influence of varying initial $\mathrm{CC}$ conditions and orbital parameters. The computations are presented in the following way. In Section 3.1 we investigate the evolution of CCs in three mass regimes (CC_H, CC_M, CC_L) of the Bastian relation. In Section 3.2 we consider a maximal model with 80 star clusters. For comparison, computations without an external gravitational field are presented in Section 3.3.

\subsection{Configurations Covering the Cluster Complex Mass Range}

We investigate the evolution of a CC configuration in three different mass regimes (Fig. 2) for circular orbits at galactic distances of $R_{\text {gal }}=2,3,5,8,12 \mathrm{kpc}$ (Section 3.1.1) and an eccentric orbit (Section 3.1.2). The number of star clusters comprising the $\mathrm{CC}$ is fixed to $N_{0}^{\mathrm{CC}}=20$ in all models. In order to get comparable starting conditions for the models CC_H, CC_M, and CC_L we introduce a parameter

$$
\alpha=\frac{R_{p l}^{S C}}{R_{p l}^{C C}},
$$

where $R_{\mathrm{pl}}^{\mathrm{SC}}$ and $R_{\mathrm{pl}}^{\mathrm{CC}}$ are the Plummer radius of a single star cluster and the Plummer radius of the CC, respectively (Fellhauer et al. 2002). The parameter $\alpha$ is a measure of how densely the CC is filled with star clusters for an equal number $N_{0}^{\mathrm{CC}}$ of star clusters. In general high values of $\alpha$ accelerate the merging process because the star clusters already overlap in the center of the CC, whereas low values hamper the merging process. We choose a medium value of $\alpha=0.08$, which leads to a reasonable range of the Plummer radii of the individual star clusters. The grids were also scaled to get the same relative resolution for all models. The chosen initial parameters are displayed in Table 1 .

\subsubsection{Simulations on Circular Orbits}

We have performed 15 simulations on circular orbits holding the initial star cluster configuration and the number of star clusters in the complex fixed. The CC masses were restricted to the high- and low-mass end (CC_H_20 and CC_L_20) of the Bastian relation and a value in-between 
(CC_M_20). The orbital parameters are given in Table 2, The circular velocities, $v_{\text {circ }}$, at the different galactic distances, $R_{\text {gal }}$, were derived from the rotation curve of NGC 1023 (Fig. 1). The orbital periods lie between 70 and 350 Myr.

The timescale of the merging process is very short. The merger object forms within a few crossing times of the CC. In all cases the merging leads to a stable final object. The properties of the merger objects at $t=5 \mathrm{Gyr}$ are displayed in Table 3 . The number of merged star clusters varies from 10 to 16 . The number increases with galactic distance as the influence of the tidal field becomes weaker.

In all cases we calculate surface density profiles and fit these by a King profile (King 1966). Three exemplary final merger objects after 5 Gyr are shown in Fig. 3 as contour plots on the $\mathrm{x}-\mathrm{y}$-plane and as corresponding surface density profiles.

The tidal radii vary from $R_{\mathrm{t}}=18.8$ pc for simulation CC_L_2_20 at $R_{\text {gal }}=2 \mathrm{kpc}$ to $R_{\mathrm{t}}=88.9$ pc for CC_H_12_20 at $R_{\text {gal }}=12 \mathrm{kpc}$. The enclosed mass of the merger objects is defined as the mass within the tidal radius. After 5 Gyr 20 to $60 \%$ of the initial CC mass is bound to the merger object. The half-mass radius is the radius of the sphere, where half of the mass is enclosed. However, as observers can only derive a projected half-mass radius, we calculate also a projected value defined as the projected radius in the surface density plot within which half of the mass is included. The projected half-mass radius is slightly smaller than the three-dimensional half-mass radius (Table 3) and corresponds to the observed effective radius, $R_{\text {eff }}$.

The enclosed masses and half-mass radii are increasing with galactic distance $R_{\text {gal }}$, leading to small compact merger objects at $R_{\text {gal }}=2 \mathrm{kpc}$ and more extended massive ones at $R_{\text {gal }}=12 \mathrm{kpc}$. Comparing the different models CC_H_20, CC_M_20, and CC_L_20 the tidal field has the largest impact on model CC_H_20 impeding the merging process. An estimate of the influence of the tidal field is given by the parameter

$$
\beta=\frac{R_{c u t}^{C C}}{R_{t}^{C C}}
$$

(Fellhauer et al. 2002), which is the fraction of the cutoff radius $R_{\text {cut }}^{\mathrm{CC}}$ of the CC and its tidal radius $R_{\mathrm{t}}^{\mathrm{CC}}$. The larger the value of $\beta$, the larger the impact. The tidal radius is estimated by using the Jacobi limit for a satellite on a circular orbit (Binney \& Tremaine 1987),

$$
\begin{aligned}
R_{t}^{C C} & =\left(\frac{M_{C C}}{3 \cdot M_{\text {gal }}\left(R_{\text {gal }}\right)}\right)^{\frac{1}{3}} \cdot R_{\text {gal }} \\
& \approx\left(\frac{M_{C C} \cdot G}{3 \cdot v_{c}^{2}\left(R_{\text {gal }}\right)}\right)^{\frac{1}{3}} \cdot R_{\text {gal }}^{\frac{2}{3}} .
\end{aligned}
$$

The values for the initial tidal radii of the CCs and the corresponding parameter $\beta$ of the individual models are shown in Table 22, The $\beta$-values are decreasing from model CC_H_20 to CC_L_20 and from small galactic distances of $R_{\text {gal }}=2 \mathrm{kpc}$ to large distances of $R_{\text {gal }}=12 \mathrm{kpc}$. Therefore model CC_H_20 suffers a higher percental mass-loss than simulations CC_M_20 and CC_L_20 and for each simulation the impact of the tidal force on the CC is larger for small galactic distances $R_{\text {gal }}$. 


\subsubsection{Cluster Complexes on an Eccentric Orbit}

We performed 3 simulations on eccentric orbits to test the influence of the orbit on the properties of the final merger object. The orbit was chosen such that the distance of the CC to the center of NGC 1023 varies between $3 \mathrm{kpc}$ and $8 \mathrm{kpc}$. The orbital period is about 100 Myr. Again the computations were performed for the three different mass regimes (Fig. 2). Table 3 shows the properties of the final merger objects.

For simulation CC_H_ecc_20 the value for the enclosed mass lies between the values of the circular orbits at $R_{\text {gal }}=2 \mathrm{kpc}$ and $R_{\text {gal }}=3 \mathrm{kpc}$. The half-mass and tidal radii agree more with those obtained for the $2 \mathrm{kpc}$ circular orbit. The properties of the final object of simulation CC_M_ecc_20 and CC_L_ecc_20 are similar to those of the circular $3 \mathrm{kpc}$ orbit. Thus, for the eccentric orbit the peri-galactic distance determines the overall properties of the final merger object.

\subsubsection{Different Initial Configurations}

For comparison we also performed simulations for two additional initial configurations in the medium mass regime (CC_M_5_c2_20 and CC_M_5_c3_20). Different seeds were used for the random number generator to generate two different initial configurations having the same input parameters as CC_M_20 (Table 1). The initial configurations vary considerable due to the low number of clusters constituting the CC. The models were placed at a distance of $R_{\text {gal }}=5 \mathrm{kpc}$ on a circular orbit.

The two configurations also lead to a fast merger within the first few crossing times. After 5 Gyr the final objects have masses of $2.5 \cdot 10^{4} \mathrm{M}_{\odot}$ (CC_M_5_c2_20) and 2.9 $\cdot 10^{4} \mathrm{M}_{\odot}$ (CC_M_5_c3_20). The corresponding projected half-mass radii are $5.5 \mathrm{pc}$ and $5.6 \mathrm{pc}$ (Table 3 ). In the case of configuration CC_M_5_c2_20 16 star clusters have merged. Whereas for configuration CC_M_5_c3_20 the number of merged star clusters is only 13 although its final mass is larger. The final mass of the merger object does not scale with the number of merged star clusters as the newly formed object looses considerable mass on its orbit around the center of NGC 1023. The final parameters of the merger objects are slightly smaller than those of the corresponding model CC_M_5_20 (see Sect. 3.1.1). While the enclosed mass and the half-mass radius of a merger object depend considerably on the initial configuration, the general order of magnitude stays the same.

\subsection{A Model With a Large Number of Star Clusters}

In this section we consider a maximal CC with $N_{0}^{\mathrm{CC}}=80$ star clusters. The parameters for the computation are presented in Table 1. The general parameters for the CCs are taken from

model CC_H_20. Due to the larger number of star clusters, the individual star clusters have lower 
masses, which are comparable to the star cluster masses of simulation CC_M_20. The complex is placed only on one circular orbit at a distance of $R_{\text {gal }}=5 \mathrm{kpc}$, because this computation is very time-consuming.

The initial star cluster distribution is displayed in Fig. 4 as a contour plot on the $\mathrm{x}-\mathrm{y}$-plane. The distribution is quite extended and has a concentration of star clusters in its center as observed for CCs in M51 by Bastian et al. (2005). Within the first 100 Myr a massive merger object forms and the unmerged star clusters align along the orbit (Fig. 5). There is still a close companion star cluster as a satellite of the merger object. The time evolution of the merger object is shown in Fig. 66 as contour plots. The snapshots were taken at $t=0.5,1,3$ and 5 Gyr. At $t=500$ Myr (top panel) the merger object has already formed. It is very extended and shows prominent tidal arms. The major mass loss occurs within the first Gyr. Later the merger object becomes more compact and its tidal arms are less pronounced. After 5 Gyr the merger object has reached an almost stable state. The mass loss is small and the tidal radius hardly changes any more.

Table 3 lists the properties of the final merger object which resulted from the merger of 32 star clusters. Its mass is only $9 \%$ of the initial CC mass and its projected half-mass radius is 8.5 pc.

\subsection{Cluster Complexes without External Gravitational Field}

For comparison we performed simulations without an external gravitational potential (i.e. $\left.R_{\mathrm{t}}=\infty\right)$ for the three models CC_H_20, CC_M_20, CC_L_20, and the maximal configuration with 80 star clusters, CC_H_80. In case of the three computations with varying CC masses 17 out of 20 star clusters merge within the first 500 Myr. After 5 Gyr nearly all star clusters have merged and formed an object with projected half-mass radii of 22.5 pc (CC_H_inf_20), 12.7 (CC_M_inf_20) and 9.0 (CC_L_inf_20) which contain more than $90 \%$ of the initial CC mass (Table 3).

For the simulation with 80 star clusters (CC_H_inf_80) the merging process is considerably slower. In the first 500 Myr 46 star clusters merge. The newly formed merger object is surrounded by several unmerged star clusters which are found preferentially in its close vicinity. During the next 500 Myr another 23 star clusters merge into the merger object. After 5 Gyr 79 star clusters have merged resulting in a final object with a projected half-mass radius of $45.6 \mathrm{pc}$ and a mass of $3.9 \cdot 10^{5} \mathrm{M}_{\odot}$ corresponding to $97.5 \%$ of the initial CC mass.

The individual star clusters in CC_H_80 have basically the same relative velocities as in CC_H_20, but four times lower masses. This reduces the probability of merging considerably. The resulting longer merging timescale provides the gravitational field more time to tear clusters off the complex. This explains the lower number of merged star clusters and the relatively low mass in the case of the corresponding model within a tidal field. 


\section{DISCUSSION AND CONCLUSION}

We summarize the major properties for all computations in Fig. 7. The models CC_H_20, CC_M_20 and CC_L_20 were placed on circular orbits at 5 different distances whereas the big configuration CC_H_5_80 with 80 star clusters and the two additional configurations were only run on a circular orbit at $R_{\text {gal }}=5 \mathrm{kpc}$. The 3 computations on an eccentric orbit are indicated by dashed lines ranging from 3 to $8 \mathrm{kpc}$. We plotted the enclosed mass and the projected half-mass radius, $R_{\text {eff }}$, against the galactic distance from the center of NGC 1023.

The enclosed masses were converted into $V$-magnitudes to allow for direct comparison with the observed data, using the formula

$$
V=M_{V, \text { solar }}-2.5 \cdot \log \left(M_{\mathrm{FF}} / x\right)+29.97,
$$

where $M_{\mathrm{V} \text {,solar }}=4.83 \mathrm{mag}$ is the absolute solar $V$ magnitude, $M_{\mathrm{FF}}$ the mass of the FF, $x$ the mass-to-light ratio and the value of 29.97 the distance modulus derived from the planetary nebula luminosity function (Ciardullo et al. 2002). We use a mass-to-light ratio of $x=2.3$, as observed by Pryor \& Mylan (1993) for 56 Globular Clusters in the Milky Way. No observational constraints on the M/L-ratio of FFs are available.

Fig. 7h shows the enclosed mass as well as the corresponding V-magnitudes versus the galactic distance $R_{\text {gal }}$. The $\mathrm{V}$-magnitudes of the merger objects cover a range from 22.4 mag to 25.6 mag. Larsen \& Brodie (2000) detected FFs in the luminosity range $21.4 \mathrm{mag}$ to 25 mag, with a median of $23.6 \mathrm{mag}$, but only one FF is brighter than $22.5 \mathrm{mag}$. The luminosities of the simulated merger objects are therefore in very good agreement with the observed luminosities of FFs.

Figure $7 \mathrm{~b}$ shows the effective radius of the merged CCs versus galactic distance. The effective radii for all simulations range between 3.6 and $13.4 \mathrm{pc}$. The effective radius criterion of $R_{\mathrm{eff}} \geq 7 \mathrm{pc}$ from Larsen \& Brodie (2000) is added as a horizontal line. The merger objects of model CC_H_20 (circular and eccentric) and the big simulation CC_H_5_80 with 80 star clusters lie clearly above the radius limit whereas the computations of model CC_M_20 exceed the limit only at galactic distances of $R_{\text {gal }} \geq 8 \mathrm{kpc}$. The lowest mass merger objects have effective radii below $7 \mathrm{pc}$. However, the effective radius criterion of $R_{\text {eff }} \geq 7 \mathrm{pc}$ is not to be taken as a definite limit but more as a reference value, because below $R_{\text {eff }}=7$ pc it is not possible to distinguish FFs from compact red GCs. $80 \%$ of the FFs observed by Larsen \& Brodie (2000) have effective radii below 14 pc. The median of the observed effective radii is 10.7 pc. Our results are therefore in good agreement with observations.

The simulations presented in this paper demonstrate that CCs from the high-mass end of the Bastian relation evolve into FF-like objects. Our formation scenario suggests that FFs are the remnants of merged CCs formed in spiral arms. NGC 1023 has a prominent bar that extents

\footnotetext{
1 Larsen \& Brodie (2000) did not consider objects below 25 mag. Their approximate $50 \%$ completeness limit was $24.5 \mathrm{mag}$.
} 
up to about $3 \mathrm{kpc}$ (Debattista et al. 2002). In barred spiral galaxies, spiral arms usually start where the bar ends (e.g. Elmegreen \& Elmegreen 1982). The absence of FFs in the inner $3 \mathrm{kpc}$ of NGC 1023 is therefore consistent with the formation scenario presented in this paper. The Milky Way, which is also a barred galaxy with a comparable size to NGC 1023, has most of its giant molecular clouds in the disk between Galactic radii 3 to $8 \mathrm{kpc}$, with a peak between 4 and $5 \mathrm{kpc}$ (Dame 1993). The mass-radius relation found for young massive CCs in M51 is comparable to the corresponding relation for giant molecular clouds in M51 (Bastian et al. 2005). Consequently, the radial distribution of FFs should in general follow the radial distribution of giant molecular clouds, but modified by the results of Fig. 7, i.e. lower masses and smaller effective radii for decreasing galactic distances. In NGC $1023 \mathrm{FFs}$ were found between projected galactic radii $3 \mathrm{kpc} \leq R_{\text {gal }} \leq$ $10 \mathrm{kpc}$, with a clear peak at a projected radius of $4.5 \mathrm{kpc}$. The observed radial distribution of FFs is therefore in good agreement with the expected distribution from our model.

In summary, the simulated merger objects based on the observed mass-radius relation from Bastian et al. (2005) resemble the observed parameters mass/luminosity, size and spatial distribution of FFs in NGC 1023 very well. The merging of young massive CCs is therefore a likely formation scenario for FFs.

The measured velocities from Brodie \& Larsen (2002) confirm the general co-rotation of the FFs with the disk of NGC 1023. Burkert et al. (2005) argued that the annular distribution of FFs on the sky and some deviations from the rotation curve of NGC 1023 indicate that the FFs form a ring-like structure with a mean radius of $5 \mathrm{kpc}$ potentially formed during a past tidal encounter. In their model, FFs are on highly eccentric orbits spanning the entire range between 3 and $8 \mathrm{kpc}$. In our model, FFs are associated with the former spiral arms of NGC 1023, as the CCs in M51 are associated with the spiral arms of the disk. FFs should therefore follow the rotation of the disk of NGC 1023, which was probably disturbed during a past interaction that transformed NGC 1023 into a lenticular galaxy. However, due to the very low luminosities, the observational uncertainties of the velocities are relatively large. Burkert et al. (2005) re-observed two FFs from Brodie \& Larsen (2002). The new measurements of these clusters yield $539 \pm 21$ and $676 \pm 13 \mathrm{~km} \mathrm{~s}^{-1}$, while the previous measurements found $514 \pm 8$ and $725 \pm 17 \mathrm{~km} \mathrm{~s}^{-1}$, respectively. These differences are too large for definite statements about deviations from circular orbits.

Brodie \& Larsen (2002) averaged all FF spectra shifted to zero velocity to achieve a high enough signal-to-noise ratio to estimate the age of these objects that appears to be older than 7-8 Gyr. They used the same integrated spectrum to derive a mean metellicity of $[\mathrm{Fe} / \mathrm{H}]=-0.58 \pm 0.24$ and a mean alpha-to-iron ratio $[\alpha / \mathrm{Fe}]$ between +0.3 and +0.6 compared to solar values. Enhanced alpha-to-iron ratios are usually associated with rapid, burst-like star formation on short time scales. Indeed, the 5 to $8 \mathrm{Myr}$ old CCs observed by Bastian et al. (2005) show a low age spread, indicating that the stellar mass of the order of $10^{5} \mathrm{M}_{\odot}$ has been formed in a burst-like event.

The FFs need to be further analyzed with respect to additional and more precise kinematical data to allow for a reliable estimate of their orbits. In addition, age estimates, metallicities, and 
alpha-to-iron ratios are needed for individual FFs. The scatter and the variation of these parameters with galactic distance will shed light on the FF evolution and further test whether the discussed formation scenario is valid.

The work of this paper was supported by DFG Grant KR 1635/14-1. We thank Søren Larsen for helpful discussions and access to their FF-data.

\section{REFERENCES}

Bastian, N., Gieles, M., Efremov, Yu. N., \& Lamers, H. J. G. L. M. 2005, A\&A, 443, 79

Binney, J., \& Tremaine, S. 1987, Galactic Dynamics (Princeton Univ. Press)

Boselli, A., Lequeux, J., \& Gavazzi, G. 2002, Ap\&SS, 281, 127

Brodie, J. P., \& Larsen, S. S. 2002, AJ, 124, 1410

Burkert, A., Brodie, J., \& Larsen, S. 2005, ApJ, 628, 231

Chies-Santos, A. L., Santiago, B. X., \& Pastoriza, M. G. 2007, A\&A, 467, 1003

Ciardullo, R., Feldmeier, J.J., Jacoby G.H., Kuzio de Naray, R., Laychak, M.B., \& Durrell P.R. 2002, ApJ, 577, 31

Dame, T.M. 1993, in AIP Conf. 278, Back to the Galaxy, ed. S. S. Holt, \& F. Verter, 267

Debattista, V.P., Corsini, E.M., \& Aguerri, J.A.L. 2002, MNRAS, 332, 65

Elmegreen, D. M., \& Elmegreen, B. G. 1982, MNRAS, 201, 1021

Fellhauer, M., Kroupa, P., Baumgardt, H., Bien, R., Boily, C. M., Spurzem, R., \& Wassmer, N. 2000, New A, 5, 305

Fellhauer, M., Baumgardt, H., Kroupa, P., \& Spurzem, R. 2002, CeMDA, 82, 113

Fellhauer, M., \& Kroupa, P. 2002, AJ, 124, 2006

Fellhauer, M., \& Kroupa, P. 2005, ApJ, 630, 879

Gieles, M., Portegies Zwart, S.F., Baumgardt, H., Athanassoula, E., Lamers, H.J.G.L.M., Sipior, M., and Leenaarts, J. 2006, MNRAS, 371, 793

Gieles, M., Athanassoula, E., Portegies Zwart, S.F. 2007, MNRAS, 376, 809

Hernquist, L. 1990, ApJ, 356, 359

King, I. R. 1966, AJ, 71, 276 
Kroupa, P. 1998, MNRAS, 300, 200

Kroupa, P. 2008, in The Cambridge N-Body Lectures, Lecture Notes in Physics, Vol. 760, eds. S. Aarseth, C. Tout, \& R. Mardling (Berlin: Springer Verlag), 181

Larsen, S. S., \& Brodie, J. P. 2000, AJ, 120, 2938

Larsen, S. S., Brodie, J. P., Huchra, J. P., Forbes, D. A., \& Grillmair, C. J. 2001, AJ, 121, 2974

Lee, M. G., Chandar, R., \& Whitmore, B. C. 2005, AJ, 130, 2128

Miyamoto, M., \& Nagai, R. 1975, PASJ, 27, 533

Noordermeer, E. et al. 2008, MNRAS, 384, 943

Peng, E.W. et al. 2006, ApJ, 639, 838

Plummer, H. C. 1911, MNRAS, 71, 460

Pryor, C., \& Meylan, G. 1993, in Structure and Dynamics of Globular Clusters, ASP Conf. Ser. 50, eds. S.G. Djorgovski, \& G. Meylan (San Francisco: ASP), 357

Salpeter E. E. 1955, ApJ, 121, 161

Simien, F., \& Prugniel, Ph. 1997, A\&AS, 126, 519

West, M.J., Côté, P., Marzke, R.O., \& Jordán, A. 2004, Nature, 427, 31 


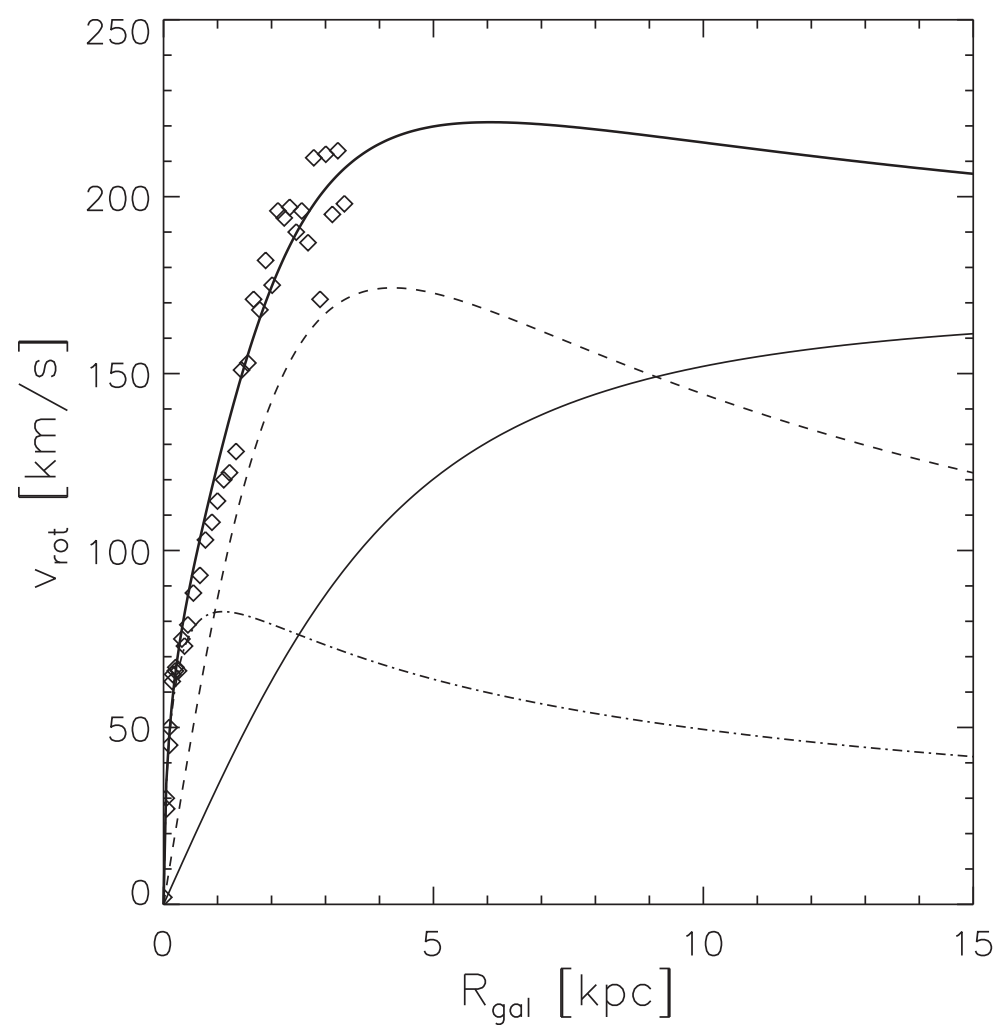

Fig. 1.- Rotation curve of a model of NGC 1023 used for the numerical simulations: the circular velocity is derived from the chosen analytical potential consisting of a Miyamoto-Nagai disk potential (dashed line), a spherical bulge potential (dashed dotted line) and a logarithmic halo (solid line). Summing up the three components yields the total potential. The diamond symbols represent the data available for the inner part (Simien \& Prugniel 1997). 


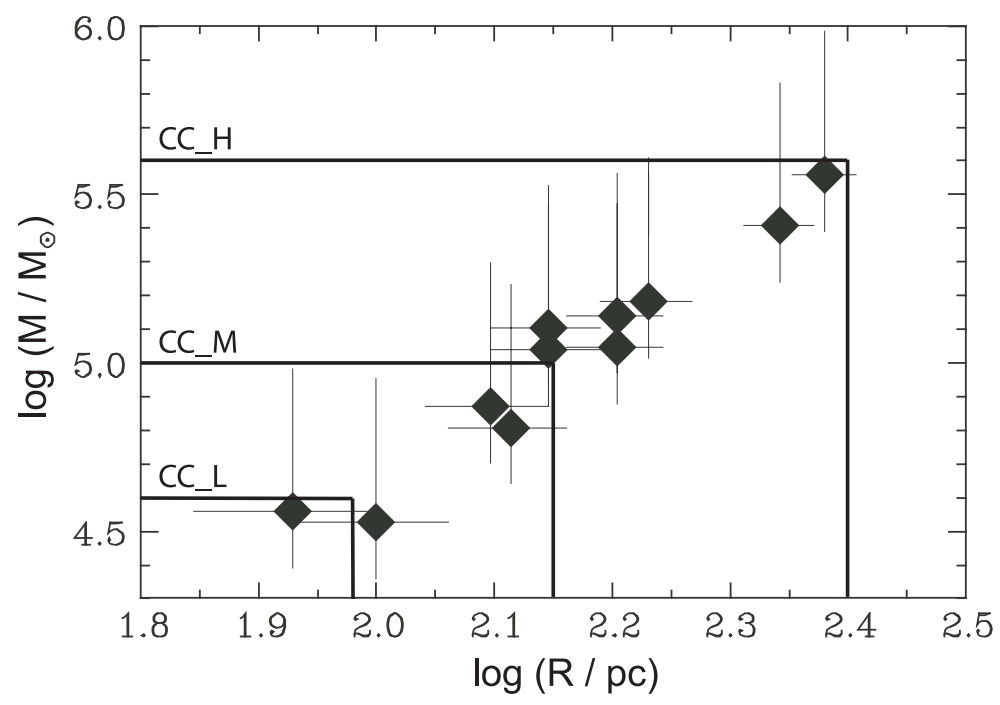

Fig. 2. - The mass versus radius relation for CCs in M51 (Bastian et al. 2005). The 11 complexes, indicated by diamonds, have sizes between $\sim 85$ and $\sim 240 \mathrm{pc}$ and cover a mass range of $0.3-3 \cdot 10^{5}$ $\mathrm{M}_{\odot}$. The size of the complex is defined as the radius where the CC is still clearly distinguishable from the background light of the galaxy. It is the cutoff radius for our models, which cover the high- and low-mass end of the Bastian relation (CC_H and CC_L) and a point in-between (CC_M). 

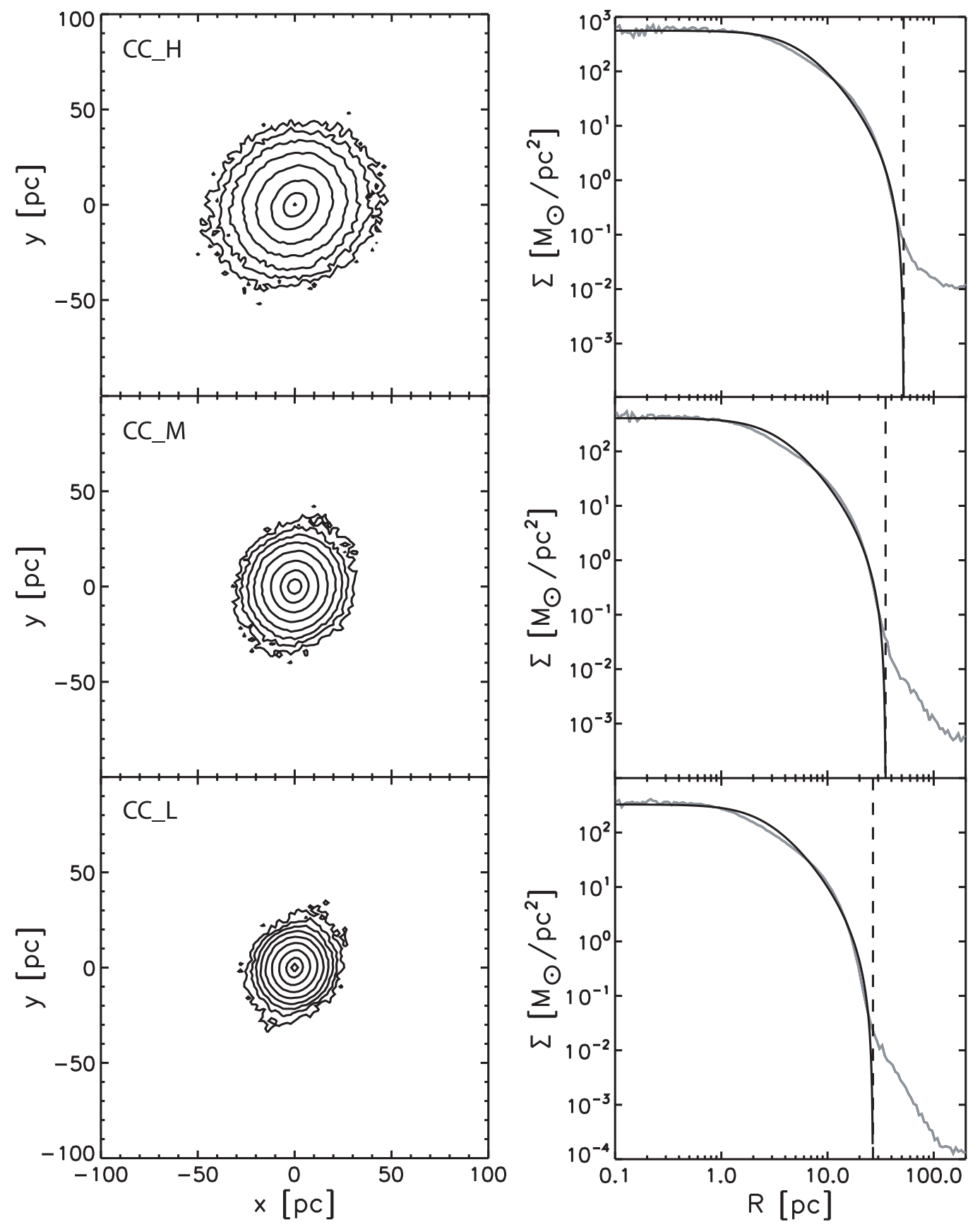

Fig. 3.- Left: Contour plots at $t=5 \mathrm{Gyr}$ on the $\mathrm{x}-\mathrm{y}$-plane for the computations CC_H_5_20, CC_M_5_20 and CC_L_5_20 at a galactic distance of $R_{\text {gal }}=5 \mathrm{kpc}$. The lowest contour level corresponds to 5 particles per pixel. The pixel size is $4 \mathrm{pc}^{2}$. This yields $0.25 \mathrm{M}_{\odot} \mathrm{pc}^{-2}$ (CC_H), $0.0625 \mathrm{M}_{\odot} \mathrm{pc}^{-2}$ (CC_M) and $0.025 \mathrm{M}_{\odot} \mathrm{pc}^{-2}$ (CC_L). The contour levels increase further by a factor of 3. Right: Surface density profile corresponding to the contour plots of CC_H_5_20, CC_M_5_20 and CC_L_5_20. The profiles are fitted by King models. The dashed vertical lines denote the tidal radii. 


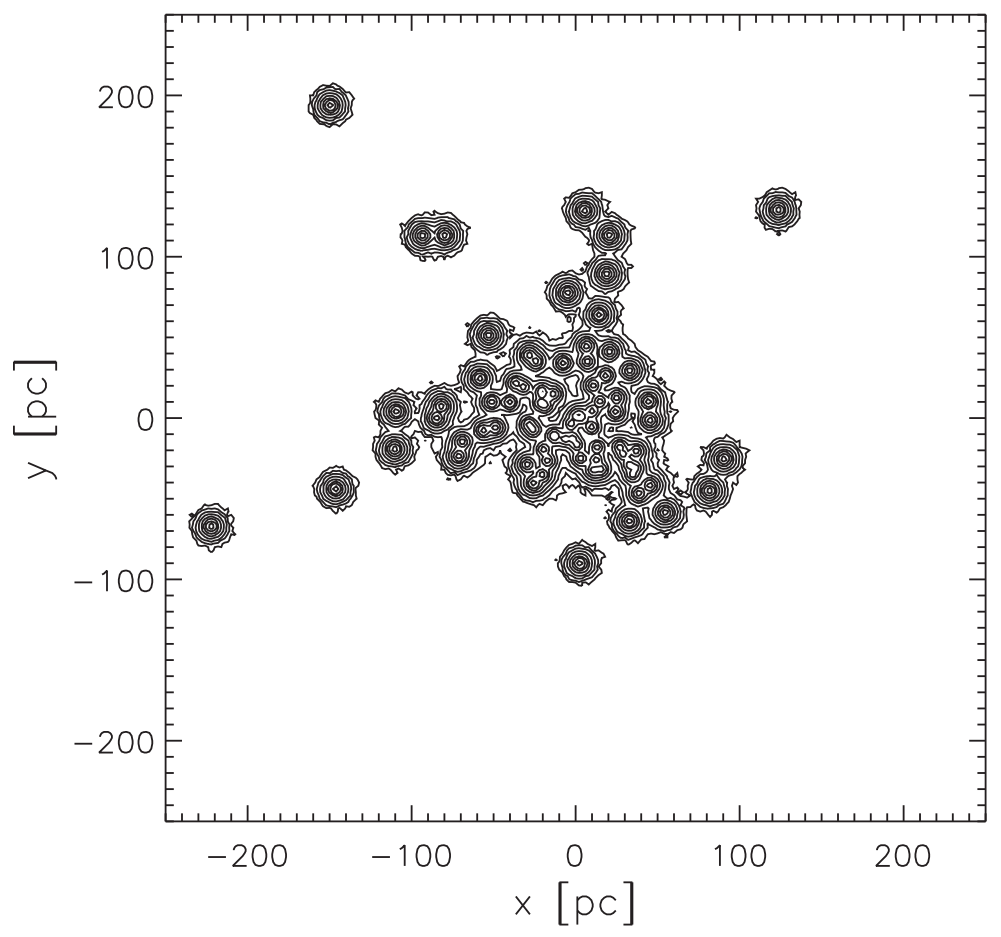

Fig. 4.- Contour plot on the $\mathrm{x}-\mathrm{y}$-plane of the initial distribution of the big computation CC_H_5_80 with 80 star clusters. The lowest contour level corresponds to 5 particles per pixel. The pixel size is $4 \mathrm{pc}^{2}$. The other contours increase by a factor of three. 


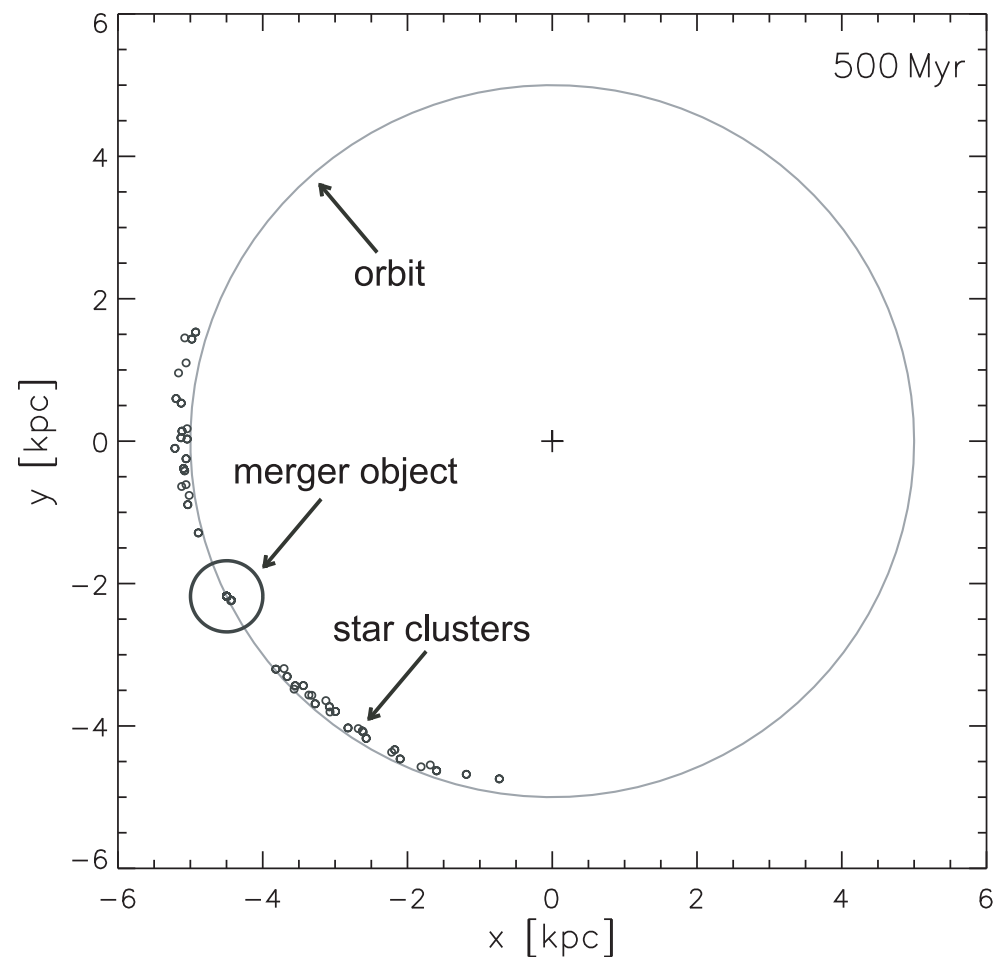

Fig. 5.- Illustration of the spatial distribution of the star clusters of computation CC_H_5_80 after 500 Myr. The CC moves on a circular orbit around the center of NGC 1023 (cross). It consists of a large merger object and a leading and trailing arm of about 50 unmerged star clusters. The circles indicate the positions but not the sizes of the star clusters. 

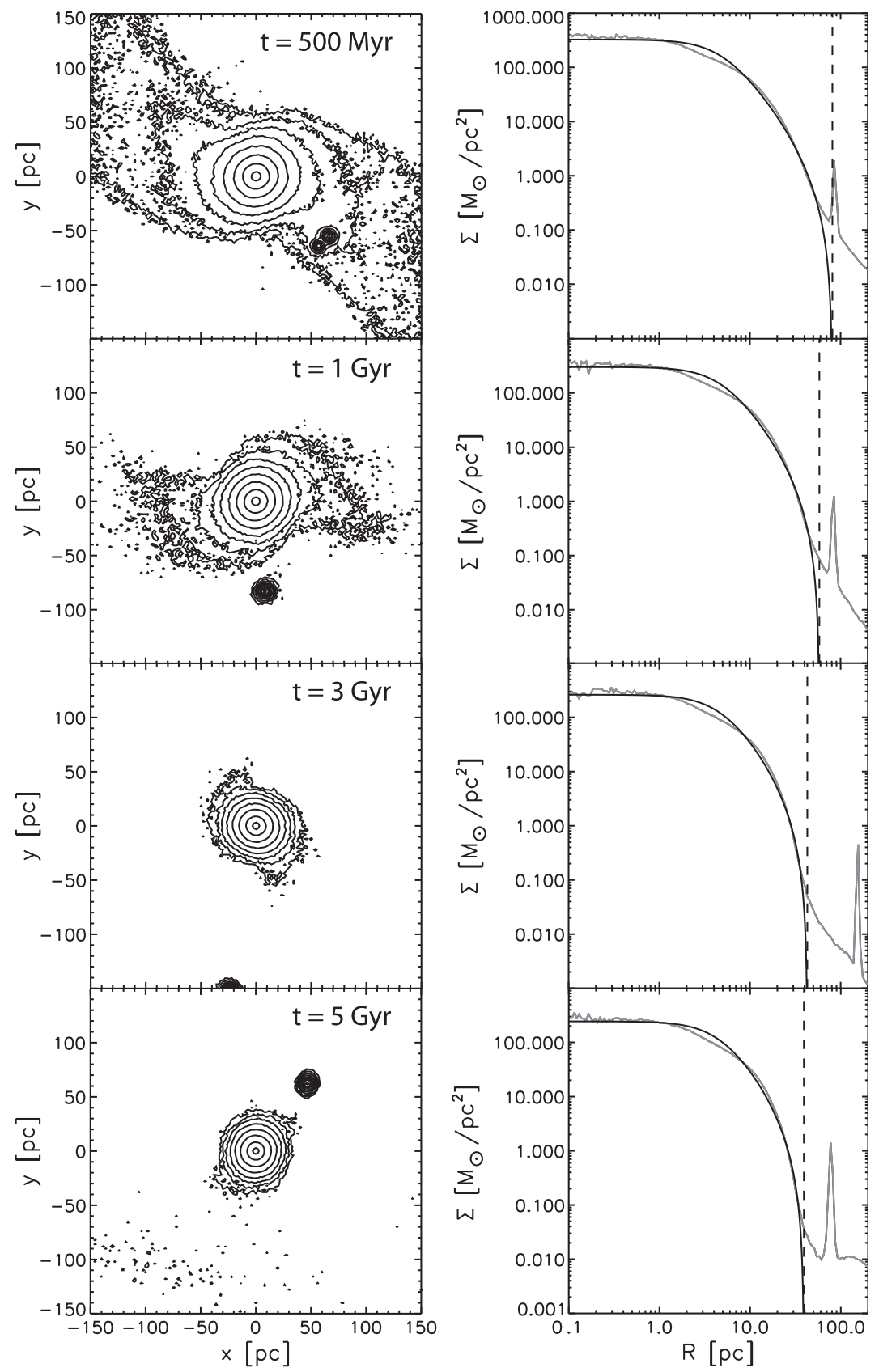

Fig. 6. - Time evolution of the merger object in simulation CC_H_5_80 at a galactic distance of $R_{\text {gal }}=5 \mathrm{kpc}$. Left: Contour plots on the x-y-plane displayed at $t=0.5,1,3,5$ Gyr. The lowest contour level corresponds to 5 particles per pixel. The pixel size is $4 \mathrm{pc}^{2}$ which corresponds to $0.0625 \mathrm{M}_{\odot} \mathrm{pc}^{-2}$. The contour levels increase further by a factor of 3 . Right: Surface density profiles corresponding to the contour plots. The profiles are fitted by King models. The dashed vertical lines denote the tidal radii. 

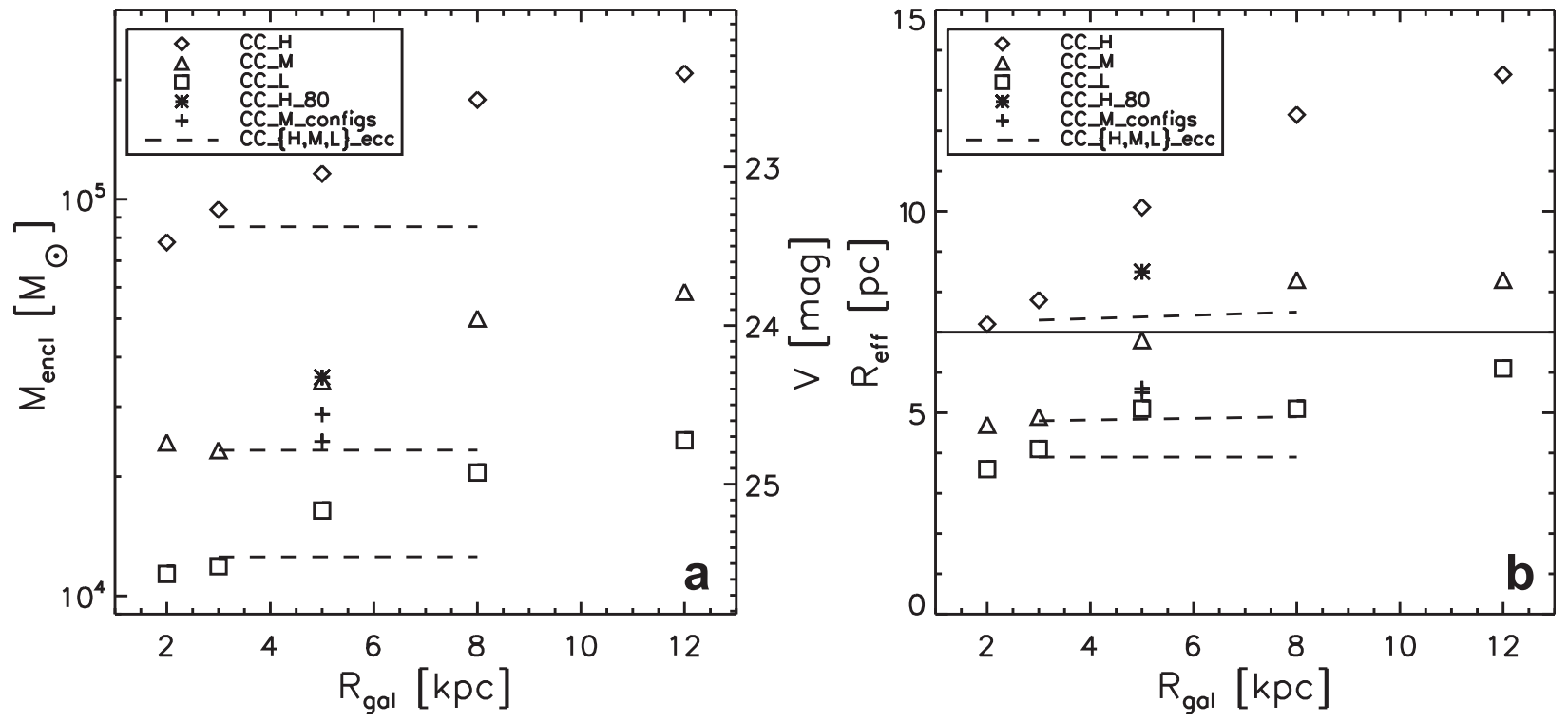

Fig. 7.- a: Enclosed mass $M_{\text {encl }}$ versus galactic distance $R_{\text {gal }}$ for all computations. Simulation CC_H_20, CC_M_20 and CC_L_20 were performed for different distances of $R_{\text {gal }}=2,3,5,8$ and 12 kpc whereas the big computation CC_H_5_80 with 80 clusters and the two additional configurations of CC_H_5_20 were only run at a distance of $R_{\text {gal }}=5 \mathrm{kpc}$. The eccentric orbit models cover a radius range of $3-8 \mathrm{kpc}$ and are therefore plotted as dashed lines. b: Effective radius $R_{\text {eff }}$ against galactic distance $R_{\text {gal }}$ for all simulations as in the left diagram. The horizontal line at $7 \mathrm{pc}$ indicates the lower size limit for FFs defined by Larsen \& Brodie (2000). 
Table 1. Initial Cluster Complex and Star Cluster Parameters

\begin{tabular}{|c|c|c|c|c|c|c|c|c|}
\hline \multirow[b]{2}{*}{ Model } & \multicolumn{4}{|c|}{ Cluster Complex } & \multicolumn{4}{|c|}{ Star Cluster } \\
\hline & $N_{0}^{\mathrm{CC} a}$ & $\begin{array}{l}M_{\mathrm{CC}}{ }^{b} \\
\left(\mathrm{M}_{\odot}\right)\end{array}$ & $\begin{array}{l}R_{\text {cut }}^{\mathrm{CC}} \\
(\mathrm{pc})\end{array}$ & $\begin{array}{l}R_{\mathrm{pl}}^{\mathrm{CCd}} \\
(\mathrm{pc})\end{array}$ & $N_{0}^{\mathrm{SC} \mathrm{e}}$ & $\begin{array}{l}M_{\mathrm{SC}}{ }^{f} \\
\left(\mathrm{M}_{\odot}\right)\end{array}$ & $\begin{array}{l}R_{\text {cut }}^{\mathrm{SC} g} \\
(\mathrm{pc})\end{array}$ & $\begin{array}{l}R_{\mathrm{pl}}^{\mathrm{SCh}} \\
(\mathrm{pc})\end{array}$ \\
\hline CC_H_20 & 20 & $4 \cdot 10^{5}$ & 250 & 50 & 100000 & $2 \cdot 10^{4}$ & 20.0 & 4.0 \\
\hline CC_H_80 & 80 & $4 \cdot 10^{5}$ & 250 & 50 & 100000 & $5 \cdot 10^{3}$ & 10.0 & 2.0 \\
\hline CC_M_20 & 20 & $1 \cdot 10^{5}$ & 141.5 & 28.3 & 100000 & $5 \cdot 10^{3}$ & 11.5 & 2.3 \\
\hline CC_L_20 & 20 & $4 \cdot 10^{4}$ & 95.5 & 19.1 & 100000 & $2 \cdot 10^{3}$ & 7.5 & 1.5 \\
\hline
\end{tabular}

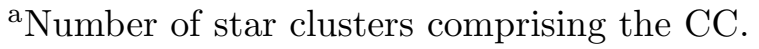

${ }^{\mathrm{b}}$ Initial CC mass.

${ }^{\mathrm{c}}$ Cutoff radius of the $\mathrm{CC}$.

${ }^{\mathrm{d}}$ Plummer radius of the $\mathrm{CC}$.

${ }^{\mathrm{e}}$ Number of star cluster particles.

${ }^{\mathrm{f}}$ Initial mass of star cluster.

${ }^{\mathrm{g}}$ Cutoff radius of star cluster.

${ }^{\mathrm{h}}$ Plummer radius of star cluster. 
Table 2. Orbital Parameters

\begin{tabular}{|c|c|c|c|c|c|c|c|c|}
\hline \multirow[b]{2}{*}{$\begin{array}{l}R_{\text {gal }}{ }^{\mathrm{a}} \\
(\mathrm{kpc})\end{array}$} & \multirow[b]{2}{*}{$\begin{array}{c}v_{\text {circ }}{ }^{\mathrm{b}} \\
\left(\mathrm{km} \mathrm{s}^{-1}\right)\end{array}$} & \multirow[b]{2}{*}{$\begin{array}{l}T_{\text {orb }}{ }^{c} \\
(\mathrm{Myr})\end{array}$} & \multicolumn{2}{|c|}{ CC_H_20 } & \multicolumn{2}{|c|}{ CC_M_20 } & \multicolumn{2}{|c|}{ CC_L_20 } \\
\hline & & & $\begin{array}{l}R_{\mathrm{t}}^{\mathrm{CCd}} \\
(\mathrm{pc})\end{array}$ & $\beta^{\mathrm{e}}$ & $\begin{array}{l}R_{\mathrm{t}}^{\mathrm{CC}} \\
(\mathrm{pc})\end{array}$ & $\beta$ & $\begin{array}{l}R_{\mathrm{t}}^{\mathrm{CC}} \\
(\mathrm{pc})\end{array}$ & $\beta$ \\
\hline 2 & 174 & 70.4 & 42.1 & 5.9 & 26.5 & 5.3 & 19.6 & 4.9 \\
\hline 3 & 202 & 91.1 & 50.0 & 5.0 & 31.5 & 4.5 & 23.2 & 4.1 \\
\hline 5 & 220 & 139.7 & 66.5 & 3.8 & 41.9 & 3.4 & 30.9 & 3.1 \\
\hline 8 & 219 & 224.3 & 91.3 & 2.7 & 57.5 & 2.5 & 42.4 & 2.3 \\
\hline 12 & 212 & 348.5 & 122.4 & 2.0 & 77.1 & 1.8 & 56.8 & 1.7 \\
\hline
\end{tabular}

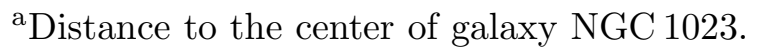

${ }^{\mathrm{b}}$ Circular velocity obtained from the rotation curve (Fig. 1).

${ }^{\mathrm{c}}$ Orbital period.

${ }^{\mathrm{d}}$ Tidal radius of CC at $t=0$ (Eq. 6).

${ }^{\mathrm{e}} \beta$-parameter at $t=0$ (Eq. 5). 
Table 3. Results of the 25 Computations after 5 Gyr

\begin{tabular}{|c|c|c|c|c|c|c|c|c|}
\hline Model & $\begin{array}{l}R_{\text {gal }}{ }^{\mathrm{a}} \\
(\mathrm{kpc})\end{array}$ & $N_{\mathrm{M}}^{\mathrm{b}}$ & $\begin{array}{l}M_{\text {encl }}{ }^{\mathrm{c}} \\
\left(\mathrm{M}_{\odot}\right)\end{array}$ & $\begin{array}{c}M_{\text {encl }} \\
(\%)\end{array}$ & $\begin{array}{c}V^{\mathrm{d}} \\
(\mathrm{mag})\end{array}$ & $\begin{array}{l}R_{\mathrm{h}}{ }^{\mathrm{e}} \\
(\mathrm{pc})\end{array}$ & $\begin{array}{l}R_{\mathrm{eff}}^{\mathrm{f}} \\
(\mathrm{pc})\end{array}$ & $\begin{array}{l}R_{\mathrm{t}}{ }^{\mathrm{g}} \\
(\mathrm{pc})\end{array}$ \\
\hline CC_H_2_20 & 2 & 10 & 77923.6 & 19.5 & 23.5 & 8.6 & 7.2 & 37.6 \\
\hline CC_H_3_20 & 3 & 12 & 94180.2 & 23.5 & 23.3 & 9.5 & 7.8 & 41.7 \\
\hline CC_H_5_20 & 5 & 12 & 116021.0 & 29.0 & 23.0 & 12.3 & 10.1 & 52.0 \\
\hline CC_H_8_20 & 8 & 14 & 178244.0 & 44.6 & 22.6 & 15.3 & 12.4 & 64.3 \\
\hline CC_H_12_20 & 12 & 15 & 207737.0 & 51.9 & 22.4 & 17.1 & 13.4 & 88.9 \\
\hline CC_H_ecc_20 & $3-8$ & 12 & 85120.6 & 21.3 & 23.4 & 9.3 & 7.4 & 38.2 \\
\hline CC_H_inf_20 & $\infty$ & 19 & 362804.0 & 90.7 & 21.8 & 29.6 & 22.5 & \\
\hline CC_H_5_80 & 5 & 32 & 35 & 8.9 & 24.3 & 10.6 & 8.5 & 39.2 \\
\hline CC_H_inf_80 & $\infty$ & 79 & 3900 & .5 & 21.7 & 60.8 & 45.6 & \\
\hline CC_M_2_20 & 2 & 12 & 2 & 4 & 24.7 & 5.7 & 4.7 & 24.4 \\
\hline CC_M_3_20 & 3 & 12 & 23297.8 & 23.3 & 24.8 & 6.0 & 4.9 & 26.4 \\
\hline CC_M_5_20 & 5 & 13 & 34793.1 & 34.8 & 24.4 & 8.3 & 6.8 & 35.3 \\
\hline CC_M_8_20 & 8 & 1 & 4 & 0.0 & 24.0 & 10.0 & 8.3 & 43.5 \\
\hline CC_M_12_20 & 12 & 16 & 584 & & 23.8 & 10.5 & 8.3 & 58.6 \\
\hline CC_M_ecc_20 & $3-8$ & 12 & 231 & 23.2 & 24.8 & 6.2 & 4.9 & 26.1 \\
\hline CC_M_5_c2_20 & 5 & 16 & 24507.4 & 24.5 & 24.7 & 6.9 & 5.5 & 29.7 \\
\hline CC_M_5_c3_20 & 5 & 13 & & 28.7 & 24.6 & 6.9 & 5.6 & 30.4 \\
\hline CC_M_inf_20 & $\infty$ & 19 & 90740.5 & 90.7 & 23.3 & 16.6 & 12.7 & - \\
\hline CC_L_2_20 & 2 & 13 & 11360.3 & 28.4 & 25.6 & 4.4 & 3.6 & 18.8 \\
\hline CC_L_3_20 & 3 & 13 & 11879.8 & 29.7 & 25.5 & 4.9 & 4.1 & 20.9 \\
\hline CC_L_5_20 & 5 & 14 & 16422.8 & 41.1 & 25.2 & 6.3 & 5.1 & 25.8 \\
\hline CC_L_8_20 & 8 & 14 & 20470.2 & 51.2 & 24.9 & 6.5 & 5.1 & 30.3 \\
\hline CC_L_12_20 & 12 & 16 & 24679.0 & 61.7 & 24.7 & 7.7 & 6.1 & 43.9 \\
\hline CC_L_ecc_20 & $3-8$ & 13 & 12491.0 & 31.2 & 25.5 & 4.8 & 3.9 & 21.1 \\
\hline CC_L_inf_20 & $\infty$ & 20 & 37643.0 & 94.1 & 24.3 & 11.7 & 9.0 & \\
\hline
\end{tabular}

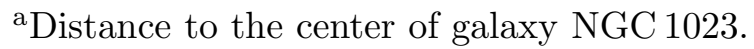

${ }^{\mathrm{b}}$ Number of merged star clusters.

${ }^{\mathrm{c}}$ Enclosed mass of merger object.

${ }^{\mathrm{d}}$ V-magnitudes (Eq. 7). 
${ }^{\mathrm{e}}$ Half-mass radius of merger object.

${ }^{\mathrm{f}}$ Effective radius, i.e. the projected half-mass radius of the merger object.

${ }^{g}$ Tidal radius of merger object obtained from King fit. 